\title{
Carcinoid tumor of the appendix: A case report
}

\author{
YUQING GU, NAICHAO WANG and HONGXING XU
}

Department of General Surgery, Taicang Hospital Affiliated to Soochow University, Taicang, Suzhou 215400, P.R. China

Received May 30, 2014; Accepted February 11, 2015

DOI: $10.3892 / 01.2015 .2997$

\begin{abstract}
Carcinoid tumors are the most common neoplasm of the appendix. The clinical presentation of these lesions is often similar to that of acute appendicitis, or the tumors are asymptomatic. The carcinoids are commonly found incidentally during histopathological examination of the resected appendix following appendectomy or other abdominal procedures. Appendiceal carcinoids usually behave as benign tumors and appendicectomy alone is a sufficient treatment in the majority of cases, while for larger lesions, right colectomy should be performed. The prognosis of patients with local appendiceal carcinoids is excellent. The present study reports the case of a 22-year-old female patient that presented with recurrent right lower abdominal pain of a three-year duration. The patient underwent successful appendectomy and recovered four days later. Subsequently, an appendiceal carcinoid tumor located at the tip of the appendix was diagnosed by histopathological examination. Follow-up examination one year after surgery revealed that the patient was well with no discomfort.
\end{abstract}

\section{Introduction}

Acute appendicitis is the most common abdominal emergency encountered by the general surgeon (1). Subsequent to diagnosis by histopathological examination, primary neoplasms of the appendix are identified in $\sim 0.5 \%$ of all surgically-removed appendices, with carcinoid tumors representing $>50 \%$ of all appendix neoplasms $(2,3)$. Carcinoid tumors arise from the neuroendocrine cells of the diffuse neuroendocrine system, which are identified in numerous locations, including the lung $(25.1 \%)$, ovaries $(0.5 \%)$, biliary system $(0.2 \%)$ and throughout the gastrointestinal tract $(73.4 \%)$ (4). Appendiceal carcinoid tumors are rare neuroendocrine neoplasms that usually behave as benign tumors, while certain lesions possess the potential for malignancy and are therefore able to metastasize (5). The probability of metastasis of appendiceal carcinoid tumors is low, $\sim 4.7 \%$ of all appendiceal carcinoid tumors (6). Lymphatic

Correspondence to: Dr Hongxing Xu, Department of General Surgery, Taicang Hospital Affiliated to Soochow University, 58 South Changsheng Road, Taicang, Suzhou 215400, P.R. China

E-mail: xuhongxing61@163.com

Key words: carcinoid tumor, appendix spread is the primary route, and hepatic metastases are rare (2). Although not a common occurrence, primary carcinoid tumors of the appendix should be considered as a cause of acute appendicitis during appendectomy. Surgical resection is recommended for the treatment of appendiceal carcinoid tumors, and the long-term prognosis of patients with appendiceal carcinoids is good (6). The present study reports the case of a 22-year-old female patient diagnosed with a carcinoid tumor located at the tip of the appendix. Written informed consent was obtained from the patient.

\section{Case report}

A 22-year-old female patient was admitted to Taicang Hospital Affiliated to Soochow University (Taicang, Suzhou, China) with the complaint of recurrent right lower abdominal pain of a three-year duration, and an additional exacerbated attack of pain that had lasted for the three days prior to presentation.

The patient reported a three-year history of recurrent dull right lower abdominal pain that had not been considered serious enough to obtain medical assistance. Three days prior to admittance, the aforementioned symptoms became aggravated and the patient presented to the Taicang Hospital Affiliated to Soochow University. Physical examination revealed a body temperature of $36.5^{\circ} \mathrm{C}$, blood pressure of $111 / 78 \mathrm{mmHg}$ and a pulse rate of 95 beats/min. During assessment, the patient experienced light direct tenderness, but no rebound tenderness, in the right lower abdomen. No palpable masses were observed in the abdomen. Laboratory tests were performed, yielding the following results: White blood cell count, $4.4 \times 10^{9}$ cells/1 (normal range, 4-10x $10^{9}$ cells/1); neutrophil proportion, $46.8 \%$ (normal range, 45-80\%); hemoglobin level, $130 \mathrm{~g} / 1$ (normal range, $110-150 \mathrm{~g} / \mathrm{l}$ ); platelet count, $177 \times 10^{9}$ platelets/l (normal range, $100-300 \times 10^{9}$ platelets/1). In addition, abdominal computed tomography revealed appendiceal inflammation, while the tumor was too small to identify (Fig. 1). The patient possessed no previous medical history and no family medical history of appendicitis.

The patient underwent surgery for the treatment of the acute attack of chronic appendicitis. The appendix was identified as $7 \mathrm{~cm}$ long and $0.8 \mathrm{~cm}$ in diameter, demonstrating hyperemia and edema. The resection of the appendix was completed without complications and the patient recovered four days later. The specimen was examined by physiological analysis and stained using hematoxylin and eosin. At the tip of the appendix, there was a well-differentiated grade I neuroendocrine tumor (Fig. 2), measuring $5 \mathrm{~mm}$ at the maximum 


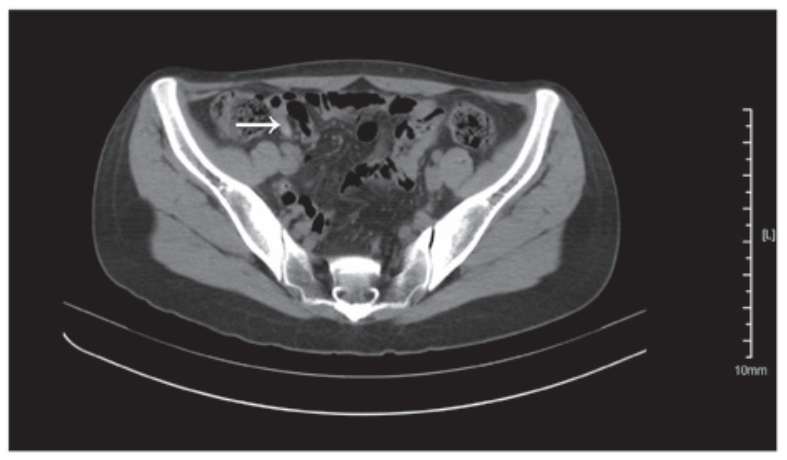

Figure 1. Computed tomography scan revealing the appendix, with the tumor being too small to identify, as indicated by the arrow.

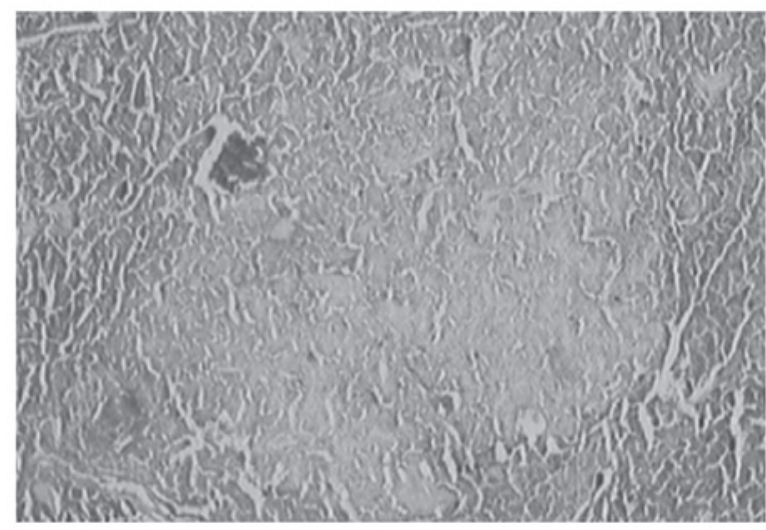

Figure 2. Photomicrograph of a hematoxylin and eosin-stained tissue section revealing the neuroendocrine tumor cells.

dimension, that had infiltrated few regions of the muscular layer. The mitotic activity of the lesion was not notable. Assessment of the Ki-67 proliferative index revealed nuclear staining in $\sim 1 \%$ of cells. The diagnosis of appendiceal carcinoid tumor was confirmed by positive immunostaining for the neuroendocrine markers chromogranin A and synaptophysin. Coexisting acute purulent appendicitis was also present. No further treatment was administered. Follow-up examination one year after surgery revealed that the patient was well, with no discomfort.

\section{Discussion}

Carcinoid tumors of the appendix are relatively uncommon neoplasms. It has been previously reported that the incidence of appendiceal carcinoids ranges between 0.3 and $0.9 \%$, as determined using histopathological examination performed on excised appendectomy specimens $(7,8)$. However, appendiceal carcinoids are the most frequent tumors arising from the appendix, comprising between 32 and $57 \%$ of all appendiceal tumors (9). Carcinoid tumors are neoplasms derived from the subepithelial neuroendocrine cells of the appendix (10) that rarely cause metastatic disease.

There is no specific pre-operative clinical presentation for appendiceal carcinoids. In general, appendiceal carcinoids are either asymptomatic or present as acute appendicitis, which is then diagnosed incidentally as appendiceal carcinoids during surgery (11). In addition, the carcinoids can result in recurrent episodes of abdominal pain due to partial obstruction of the appendiceal lumen by a tumor. The presence of neuroendocrine symptoms, including flushing, diarrhea and cardiac disease, are rarely reported (12). In the case of the present patient, the tumor was located at the tip of the appendix, and the patient experienced recurrent right lower abdominal pain that had been present for three years. The surgical procedure was performed for the treatment of appendicitis, and the carcinoid tumor was identified incidentally during the histological examination of the excised surgical specimen.

Appendiceal carcinoids grow slowly, and the overall prognosis is excellent (13). At present, tumor size is the most reliable indicator for the assessment of the malignant potential of lesions. In the majority of patients with appendiceal carcinoids, the tumor diameter is $<1 \mathrm{~cm}$ and appendectomy alone is a sufficient treatment as carcinoids rarely metastasize. However, tumors measuring $\geq 2 \mathrm{~cm}$ in diameter may possess the potential for metastasis, and patients with tumors of this size require right hemicolectomy. For tumors measuring 1-2 $\mathrm{cm}$ in diameter, the surgical options depend on mesoappendiceal involvement and the histological subtype of the lesion. Suitable candidates for right hemicolectomy include lesions where there is histological evidence of mesoappendiceal extension, tumors that are located at the base of the appendix with positive margins or involvement of the caecum, and high-grade malignant carcinoids $(14,15)$. In the present patient, the tumor was $0.5 \mathrm{~cm}$ in diameter and therefore appendectomy alone was performed. 
Post-operative follow-up was recommended by the National Comprehensive Cancer Network 2013 Guidelines following the excision of appendiceal carcinoids in for patients with tumors that are $>2 \mathrm{~cm}$ in diameter. This follow-up procedure comprises a history and physical examination every 3-12 months post-resection and every 6-12 months thereafter for $\leq 10$ years, with consideration of follow-up imaging or laboratory markers, such as 5-hydroxyindoleacetic acid or chromogranin A (16).

Overall, the long-term prognosis of appendiceal carcinoids is extremely good. Sandor and Modlin evaluated 1570 carcinoid tumors of the appendix that were treated at the American National Cancer Institute between 1973 and 1991. It was reported that the five-year survival rates of patients with localized, regional metastases and distant metastases in appendiceal carcinoids were 94.0, 84.6 and $33.7 \%$, respectively, and the overall five-year survival rate was $85.9 \%$ (9). Another study with a large cohort performed by Modlin et al reported that patients with local disease demonstrate a five-year survival rate of $92 \%$, those with regional metastases demonstrated a five-year survival rate of $81 \%$ and the few with distant metastases demonstrated a five-year survival rate of $31 \%$ (10).

Carcinoid tumors are the most common neoplasms of the appendix, but demonstrate no specific clinical presentation. The presence of these tumors should be considered, particularly during surgical procedures.

\section{References}

1. Ruffolo C, Fiorot A, Pagura G, Antoniutti M, Massani M, Caratozzolo E, Bonariol L, Calia di Pinto F and Bassi N: Acute appendicitis: what is the gold standard of treatment? World J Gastroenterol 19: 8799-8807, 2013.
2. Deans GT and Spence RA: Neoplastic lesions of the appendix. Br J Surg 82: 299-306, 1995.

3. Pickhardt PJ, Levy AD, Rohrmann CA Jr and Kende AI: Primary neoplasms of the appendix manifesting as acute appendicitis: CT findings with pathologic comparison. Radiology 224: 775-781, 2002.

4. Modlin IM and Sandor A: An analysis of 8305 cases of carcinoid tumors. Cancer 79: 813-829, 1997.

5. Parkes SE, Muir KR, al Sheyyab M II, Cameron AH, Pincott JR, Raafat $F$ and Mann JR: Carcinoid tumours of the appendix in children 1957-1986: Incidence, treatment and outcome. $\mathrm{Br}$ J Surg 80: 502-504, 1993.

6. Moertel CG, Weiland LH, Nagorney DM and Dockerty MB: Carcinoid tumor of the appendix: treatment and prognosis. N Eng J Med 317: 1699-1701, 1987.

7. Moertel CG, Dockerty MB and Judd ES: Carcinoid tumors of the vermiform appendix. Cancer 21: 270-278, 1968.

8. Connor SJ, Hanna GB and Frizelle FA: Appendiceal tumors: Retrospective clinicopathologic analysis of appendiceal tumors from 7,970 appendectomies. Dis Colon Rectum 41: 75-80, 1998.

9. Sandor A and Modlin IM: A retrospective analysis of 1570 appendiceal carcinoids. Am J Gastroenterol 93: 422-428, 1998.

10. Modlin IM, Lye KD and Kidd M: A 5-decade analysis of 13,715 carcinoid tumors. Cancer 97: 934-959, 2003.

11. Roggo A, Wood WC and Ottinger LW: Carcinoid tumors of the appendix. Ann Surg 217: 385-390, 1993.

12. Markgraf WH and Dunn TM: Appendiceal carcinoid with carcinoid syndrome. Am J Surg 107: 730-732, 1964.

13. Thirlby RC, Kasper CS and Jones RC: Metastatic carcinoid tumor of the appendix. Report of a case and review of the literature. Dis Colon Rectum 27: 42-46, 1984.

14. Syracuse DC, Perzin KH, Price JB, Wiedel PD and MesaTejada R: Carcinoid tumors of the appendix. Mesoappendiceal extension and nodal metastases. Ann Surg 190: 58-63, 1979.

15. Gouzi JL, Laigneau P, Delalande JP, Flamant Y, Bloom E, Oberlin P and Fingerhut A; The French Associations for Surgical Research: Indications for right hemicolectomy in carcinoid tumors of the appendix. Surg Gynecol Obstet 176: 543-547, 1993.

16. National Comprehensive Cancer Network (NCCN): NCCN Clinical Practice Guidelines v2.2013. Neuroendocrine tumors. http://www.nccn.org/professionals/physician_gls/pdf/neuroendocrine.pdf Accessed May 15, 2013. 